\title{
Time-domain astronomy with the Fermi GBM
}

\author{
C. M. Hui* \\ NASA/MSFC \\ on behalf of the Fermi-GBM Team \\ E-mail: c.m.hui@nasa.gov
}

The Fermi Gamma-ray Burst Monitor (GBM) is an all-sky monitoring instrument sensitive to energies from $8 \mathrm{keV}$ to $40 \mathrm{MeV}$. Over the past 8 years of operation, the GBM has detected over 240 gamma-ray bursts per year and provided timely GCN notices with localization to few-degree accuracy for follow-up observations. In addition to GRBs, Galactic transients, solar flares, and terrestrial gamma-ray flashes have also been observed. In recent years we have also been searching the continuous GBM data for electromagnetic counterpart to astrophysical neutrinos and gravitational wave events, as these are believed to be associated with gamma-ray bursts. With continuous data downlink every few hours and a temporal resolution of 2 microseconds, GBM is well suited for observing transients and supporting EM followup in the era of multi-messenger astronomy.

35th International Cosmic Ray Conference - ICRC2017

10-20 July, 2017

Bexco, Busan, Korea

${ }^{*}$ Speaker. 


\section{The GBM instument}

The Gamma-ray Burst Monitor (GBM) is an instrument onboard the Fermi Gamma-ray Space Telescope designed to monitor the entire sky for transients. It consists of $12 \mathrm{NaI}$ detectors and 2 BGO detectors, covering a field of view of $>8$ steradians and sample the entire sky every $\sim 90$ minutes. The NaI detectors are sensitive to energies from $8 \mathrm{keV}$ to $1 \mathrm{MeV}$. The $2 \mathrm{BGO}$ detectors are sensitive to energies from $200 \mathrm{keV}$ to $40 \mathrm{MeV}$. For more technical details, please see [1].

\section{Science}

Since launching in 2008, GBM has detected over 2000 gamma-ray bursts (GRBs), $\sim 2$ every 3 days. Each year, GBM detects $\sim 200$ long GRBs and $\sim 40$ short GRBs. The 3rd GRB catalog [2] summarizes the first 6 years of operations and characterize the 1405 GRB events seen by GBM. The spectral properties of the first 4-years of GRBs are described in the time resolved spectral catalog [3]. The 8-year spectral catalog is currently in preparation. In addition to GRBs, GBM also triggers on Galactic sources, terrestrial gamma-ray flashes, and solar flares, accounting for $\sim 44 \%$ of all triggers in the first 6 years. Figure 1 shows the distribution of trigger classifications and sky positions from the 6-year catalog.
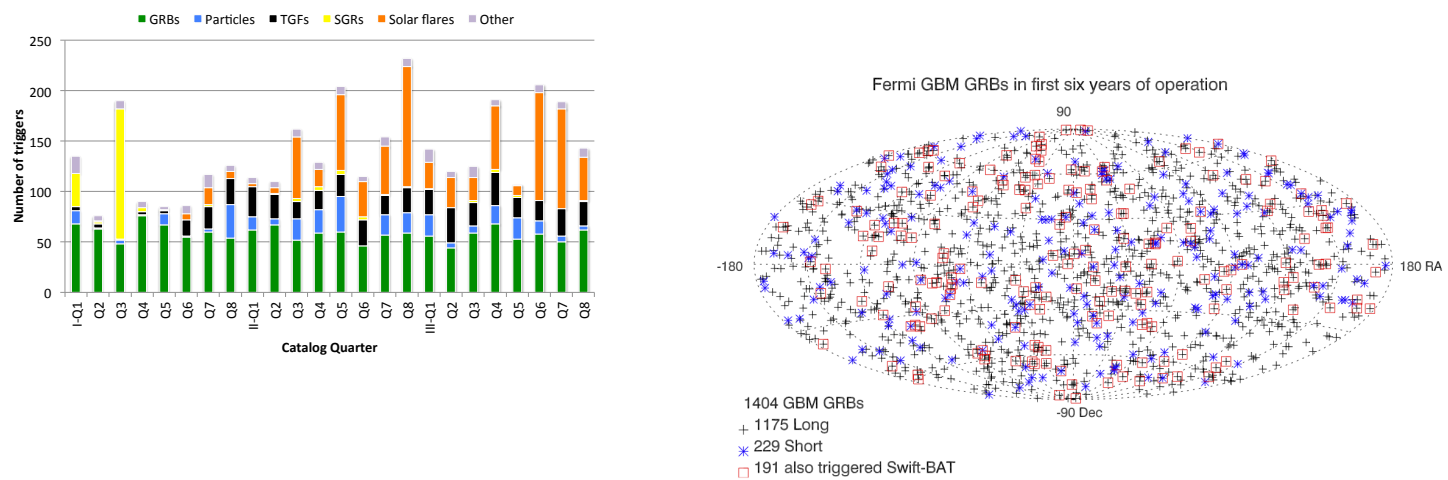

Figure 1: Left: Trigger statistics from the first 6 years of GBM operation binned quarterly. Right: Sky distributions of GRBs detected by GBM in the first 6 years, short and long are categorized by the $T_{90}$ and red squares highlight joint detections with Swift.

Burst-like events within our Galaxy has also been observed by GBM. Between 2010 and 2013, 1084 X-ray bursts have been detected and are described in the 3-year catalog [4]. Source classification is done via spectral analysis and localization, which further divided the sample into thermonuclear X-ray bursts, accretion flares and X-ray pulses, and untriggered GRBs. Thermonuclear burst rate after duty cycle correction is 1.4 per day at a distance of $\leq 10 \mathrm{kpc}$. GBM observation of these bursts provided an estimate of mass ejections and constrain the contribution to interstellar medium in our Galaxy.

With the all-sky coverage, GBM can monitor known pulsars for outbursts and also search for pulsed sources. Accreting pulsars are detected by their frequency modulation, and long term 
observations help determine the characteristics of the orbit and the neutron star. Currently 8 persistent pulsars and 28 transient pulsars are monitored and the results are available on our website https://gammaray.nsstc.nasa.gov/gbm/science/pulsars.html.

GBM also detects terrestrial gamma-ray flashes (TGFs), intense and short (millisecond timescale) gamma rays produced in Earth's atmosphere. TGFs are associated with electrons accelerating in electric fields at the top of thunderstorm clouds. These events are present in the triggered data and also from offline search [5]. The online catalog contains 4144 TGFs in over 8 years of data and is accessible at https://fermi.gsfc.nasa.gov/ssc/data/access/gbm/tgf.

Additional science not discussed here are soft gamma-ray repeaters and solar flares, more information is available at https://gammaray.nsstc.nasa.gov/gbm.

\section{Techniques}

Analysis development to increase the detection rate of GRBs in GBM data have been ongoing. Currently there are two algorithms searching for faint events in the data that did not trigger GBM by using the continuous time-tagged events (CTTE), which has $2 \mu$ s resolution and 128 energy channels: the untargeted search which looks for signal excess in 2 detectors as the offline data becomes available, and the targeted search which looks for a coherent signal in all 14 detectors when given a candidate time. These methods are also useful for multi-wavelength and multi-messenger followup and joint studies, such as counterparts to neutrinos and gravitational waves. Additionally, the Earth occultation technique is also utilized in electromagnetic counterpart searches.

\subsection{Untargeted search}

Based on the search algorithm developed for terrestrial gamma-ray flashes, this search looks for signals in $2 \mathrm{NaI}$ detectors with $2.5 \sigma$ and $1.25 \sigma$ above background in the continuous time-tagged events. Additionally the 2 detectors must have valid geometry for a point source. The search goes through 18 timescales from 64ms to 32s, and 4 energy ranges that are optimized for short GRBs. A one-day Poisson probability is calculated for each event and a threshold for notice is established at 1e-6. Figure 2 shows the probability distributions of candidates with positive and negative excesses. Verification with other instruments such as Swift and INTEGRAL are under way. Figure 3 shows a candidate found that corresponds to Swift GRB 140606A which did not trigger GBM onboard.

Currently a list of candidates since 2013 are available at our website http://gammaray.nsstc.nasa.gov/gbm/science/sgrb_search.html. GCN notices for subthreshold events found by this search will become available to the public this summer. Localization FITS file, contour sky map, and the lightcurve will accompany the GCN notices. The time delay for the notice publication will range from 0.5 to 6 hours, due to ground processing of batched downlinked data. The expected rate of notice will be $\sim 70$ per month, during periods of galactic transients activity the rate is expected to be higher. More information will be available at $h t t p: / / g c n . g s f c . n a s a . g o v / g c n / f e r m i . h t m l$.

\subsection{Targeted search}

This algorithm utilizes the continuous time-tagged events and look for coherent signal in all 14 detectors when given a time, and an optional sky map. The development was motivated by the compact binary mergers that produce gravitational waves can also produce short GRBs. This 


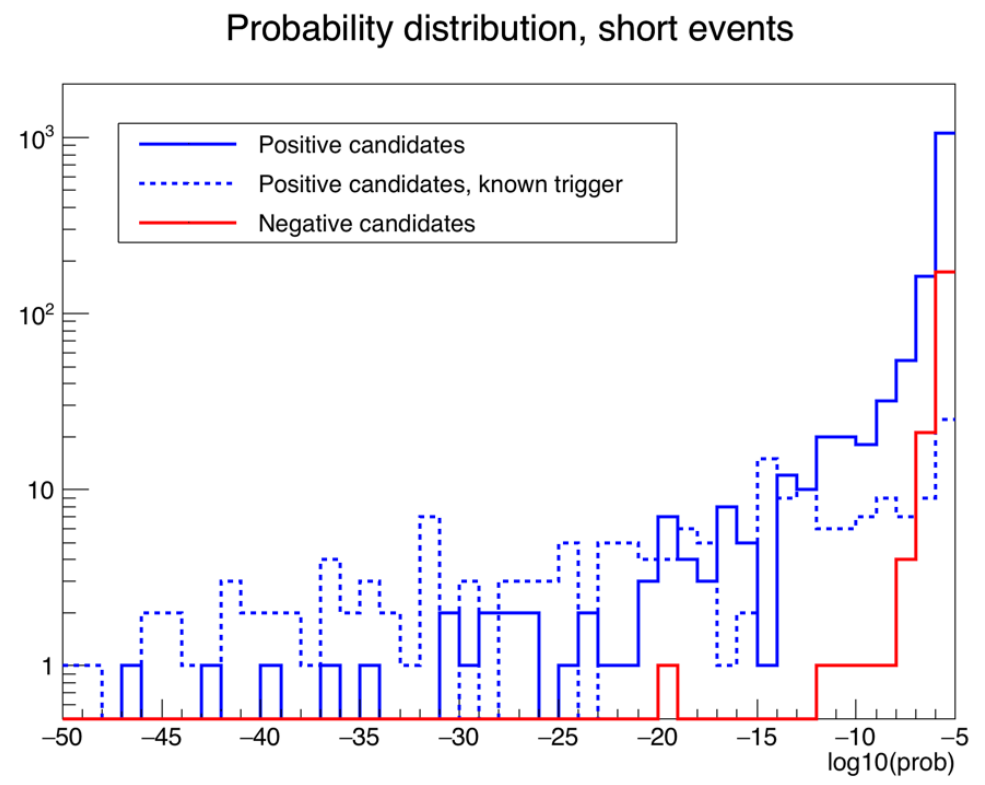

Figure 2: Probability distribution of short duration candidates with positive and negative signal excesses in 30 months of data. The current probability threshold of 1E-6 is established based on negative candidates arising from signal fluctuations.

method calculates a likelihood ratio comparing expected and observed counts in each of the 14 detectors for 3 spectral templates, and uses a uniform spatial prior unless a sky map is provided [6]. The current targeted search pipeline look for events within 30s of input time, in timescales from $0.256 \mathrm{~s}$ to $8.192 \mathrm{~s}$ with a factor of 4 phase shift for each timescale, but is capable of going down to 0.064s. Details of the current targeted search is documented in [7], along with verification using data with triggered short GRBs, background with no known source, and GRB simulation.

\subsection{Occultation}

The Earth occultation technique measures the flux change from the source entering or exiting Earth occultation. It uses continuous high time resolution (CTIME) data which has $0.256 \mathrm{~s}$ time resolution and 8 energy channels. A catalog of $200+$ sources that are being monitored can be found at the website https://gammaray.nsstc.nasa.gov/gbm/sciencelearth_occ.html. For each source, daily lightcurve in FITS and ASCII formats are available. The occultation monitoring technique detects 102 of the monitored sources, with 9 of them above $100 \mathrm{keV}$. The Crab Nebula is observed to undergo flux variations on the order of $10 \%$ on average and up to $40 \%$ at $300-500 \mathrm{keV}$ [8].

Most recent result from the occultation technique is the blackhole binary system V404 Cyg outbursts [9]. It underwent an active period over 13 days, reaching a flux level of 30x Crab flux. The occultation technique is used for follow-up observation as well.

\section{Summary}

The Fermi Gamma-ray Burst Monitor is in its 9th year of operation and continues to be prolific in detecting GRBs. With the implementation of additional offline data searches, we expect to 

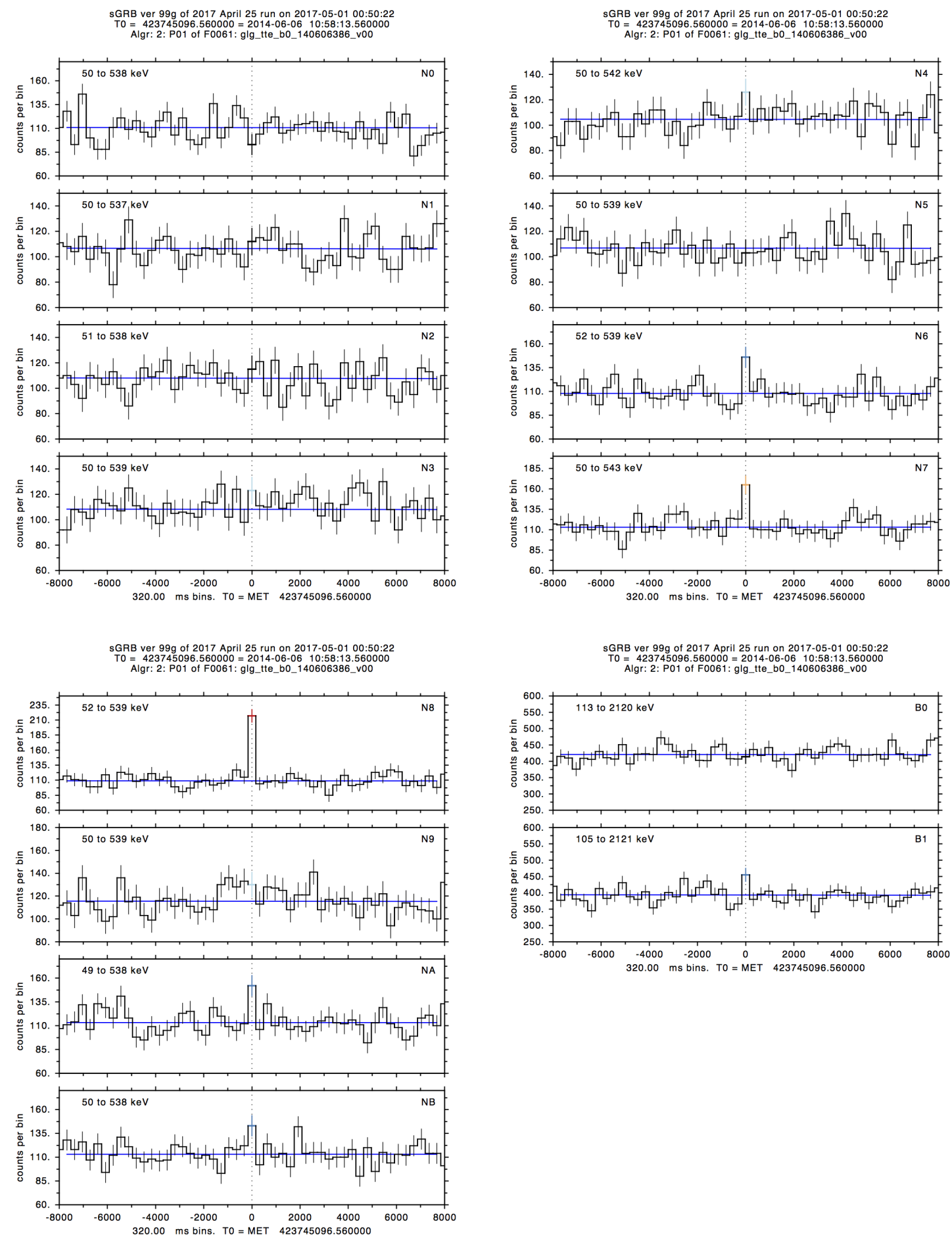

Figure 3: Individual detector lightcurves of the candidate event found by the untargeted search coincident with Swift GRB 140606A. 
increase the detection rate of short GRBs by two-fold, to $\sim 80$ per year. These search techniques also increase our capability to follow up on detections from other instruments such as IceCube and LIGO/VIRGO in the search of gamma-ray counterparts to neutrinos, gravitational waves, and other transients.

\section{References}

[1] Meegan, C., Lichti, G., Bhat, P. N., et al. 2009, ApJ, 702, 791-804

[2] Narayana Bhat, P., Meegan, C. A., von Kienlin, A., et al. 2016, ApJS, 223, 28

[3] Yu, H.-F., Preece, R. D., Greiner, J., et al. 2016, A\&A, 588, A135

[4] Jenke, P. A., Linares, M., Connaughton, V., et al. 2016, ApJ, 826, 228

[5] Briggs, M. S., Xiong, S., Connaughton, V., et al. 2013, Journal of Geophysical Research (Space Physics), 118, 3805

[6] Blackburn, L., Briggs, M. S., Camp, J., et al. 2015, ApJS, 217, 8

[7] Goldstein, A., Burns, E., Hamburg, R., et al. 2016, arXiv:1612.02395

[8] Wilson-Hodge, C. A., Cherry, M. L., Case, G. L., et al. 2011, ApJL, 727, L40

[9] Jenke, P. A., Wilson-Hodge, C. A., Homan, J., et al. 2016, ApJ, 826, 37 